Vietnam Journal of Mechanics, VAST, Vol.40, No. 4 (2018), pp. $407-424$

DOI: https://doi.org/10.15625/0866-7136/12294

\title{
COMPARATIVE STABLE WALKING GAIT OPTIMIZATION FOR SMALL-SIZED BIPED ROBOT USING META-HEURISTIC OPTIMIZATION ALGORITHMS
}

\author{
Tran Thien Huan ${ }^{1}$, Ho Pham Huy Anh ${ }^{2, *}$ \\ ${ }^{1}$ Ho Chi Minh City University of Technology and Education (HCM-UTE), Vietnam \\ ${ }^{2}$ Ho Chi Minh City University of Technology, VNU-HCM, Vietnam \\ *E-mail: hphanh@hcmut.edu.vn \\ Received April 19, 2018
}

\begin{abstract}
This paper proposes a new way to optimize the biped walking gait design for biped robots that permits stable and robust stepping with pre-set foot lifting magnitude. The new meta-heuristic CFO-Central Force Optimization algorithm is initiatively applied to optimize the biped gait parameters as to ensure to keep biped robot walking robustly and steadily. The efficiency of the proposed method is compared with the GA-Genetic Algorithm, PSO-Particle Swarm Optimization and Modified Differential Evolution algorithm (MDE). The simulated and experimental results carried on the prototype small-sized humanoid robot demonstrate that the novel meta-heuristic CFO algorithm offers an efficient and stable walking gait for biped robots with respect to a pre-set of foot-lift height value.
\end{abstract}

Keywords: biped robot, meta-heuristic optimization algorithm, Central Force Optimization (CFO) algorithm.

\section{INTRODUCTION}

The walking gesture of human up to now still contains many such sophisticated concepts that the humanoid robot can not fully demonstrate. Thus the research for biped robot walking mechanism is being developed in different directions. Several standards have been applied to human robots to ensure stable and natural gait. Static walking is the first applied principle, in which the vertical projection of center of mass (COM) of the ground is always in the supporting foot. In other words, humanoid robots can stop at any times when walking without falling apart. By its very nature, this principle applies to slow-speed robots whereby dynamic effects can be ignored [1,2]. Researchers then began to focus on developing dynamic walking [3]. This method allows the human robot to speed up the pace. However, during the robot process, the robot may fall due to environmental interference and can not stop abruptly. Therefore, ZMP-based walking is proposed to help control and manipulate inertia [4,5].

(C) 2018 Vietnam Academy of Science and Technology 
Recently, several studies have focused on improving the performance of humanoid robot walking gesture. Huang in [6] introduces a stable gait using the gaiter to use the interpolation function. The method developed by D. Huan, through the GA algorithm, optimizes the gait generator to help robot move steadily with the least amount of energy [7]. Dip et al. [8] exhibit steady gait at constant velocity using the sine wave generator. Maximo et al. [9] introduced a new stable and fast model-free gait with arms movement for humanoid robots. Khusainov et al. (2018) [10] successfully combined kinematic and dynamic approaches in gait optimization for humanoid robot locomotion. Intelligent algorithms are applied in this method to optimize the gait generator for humanoid robots such as genetic algorithm (GA) [8], algorithm for optimal swarm (PSO) [11], modified differential algorithm (MDE) [12,13]. Shaffi in [14] introduces the humanoid robot achieved a stable gait by using the Fourier series gait generator. These methods used intelligent algorithms to develop human robot walking movement, such as bee swarm algorithm [15], fuzzy TS controller [16], evolutionary technique [17,18], Ant-Colony optimization (ACO) optimized by recurrent neural networks [19], Pontryagin's maximum principle [20] and so on. Among various meta-heuristic approaches, the powerful potential of the CFOCentral Force Optimization algorithm has not yet being applied to optimize the biped robot walking gait generator.

To overcome this gap, the paper proposes a robot outlier based on the Central Force Optimization (CFO) algorithm, based on the dynamic walking method, and use ZMP Standard to maintain stability. The simulation and empirical results show that using the CFO algorithm allows optimal optimization of the gait parameters for the robot to reach steady gait with accurate foot lifting magnitude. Small-sized humanoid robot HUBOT-5 is used to verify the experimental results.

The rest of this paper is arranged as follows. Section 2 introduces the original HUBOT5 biped robot. Section 3 presents the new stable gait generation fot small-sized biped robot HUBOT-5. Section 4 proposes the novel gait parametric optimization using CFO technique. Section 5 presents and analyses the simulation and experiment results. Finally conclusion is presented in Section 6.

\section{HUMANOID ROBOT MODEL}

Small-sized humanoid robot (HUBOT-5) consists of the upper torso and two legs as described in Fig. 1. Each leg consists of 3 parts, which are femoral, legs, and foot with a total of $6 \mathrm{DOF}$ (degree of freedom), including $3 \mathrm{DOF}$ at the hip, 1 at the knee and 2 at the foot. The HUBOT-5 can mimic the walking gesture of human with respect to the front size interface (YZ - Frontal view) and the side view (XZ - Sagittal view). Total weight of the HUBOT-5 is about $1.5 \mathrm{~kg}$, including the dynamic actuator, sensor, controller, amplifier and it has a height of about $50 \mathrm{~cm}$. The HUBOT-5 is innovatively designed to ensure full dynamic structure, with each dof is corresponding with 1 independent actuator. The Servo DC engine HD-1501 is used as the actuator. The significant advantage of HD-1501 servo motor is small, compact and light (60 g) with twisted momentum 17 $\mathrm{kg}$. The control signal supplied to the servo via MATLAB/Simulink using the RS-485 transmission standard. 


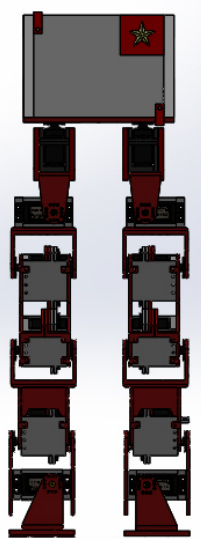

(a)

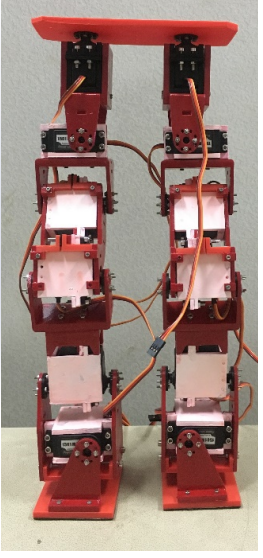

(b)

Fig. 1. HUBOT-5 humanoid robot set-up with $12 \mathrm{DOF}$

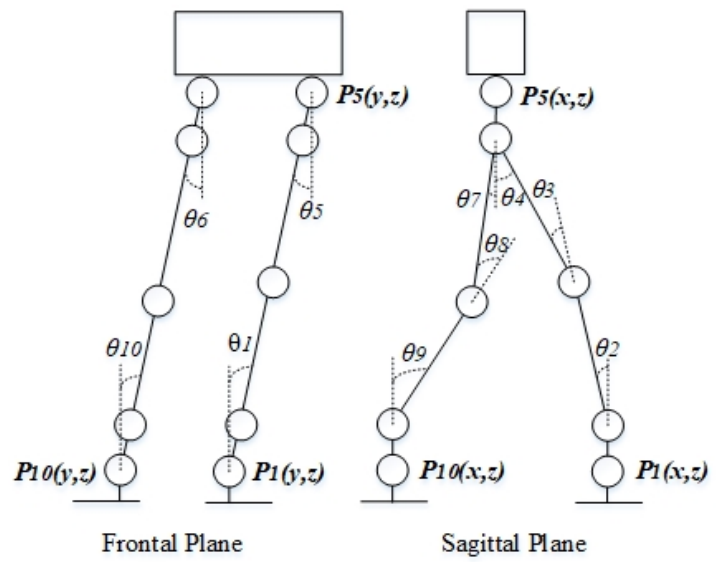

Fig. 2. Humanoid HUBOT-5 structure

This paper focuses on the straight walking gait parametric optimization of the humanoid robot, as the upper torso is fixated and then only the 10 engines with respect to ten DOF are being controlled and defined as presented in Fig. 2. The bounds of these 10 rotary angles depend on the real set-up of the HUBOT-5 biped and are tabulated in Tab. 1.

Table 1. Ten angular limitations

\begin{tabular}{ccccc}
\hline Angle & Plane & Leg & Joint & Value \\
\hline$\theta_{1}$ & $\mathrm{YZ}$ & Right & Ankle & $-20^{\circ}$ to $20^{\circ}$ \\
$\theta_{2}$ & $\mathrm{XZ}$ & Right & Ankle & $-30^{\circ}$ to $30^{\circ}$ \\
$\theta_{3}$ & $\mathrm{XZ}$ & Right & Knee & $-30^{\circ}$ to $30^{\circ}$ \\
$\theta_{4}$ & $\mathrm{XZ}$ & Right & Hip & $-30^{\circ}$ to $30^{\circ}$ \\
$\theta_{5}$ & $\mathrm{YZ}$ & Right & Hip & $-20^{\circ}$ to $20^{\circ}$ \\
$\theta_{6}$ & $\mathrm{YZ}$ & Left & Hip & $-20^{\circ}$ to $20^{\circ}$ \\
$\theta_{7}$ & $\mathrm{XZ}$ & Left & Hip & $-30^{\circ}$ to $30^{\circ}$ \\
$\theta_{8}$ & $\mathrm{XZ}$ & Left & Knee & $-30^{\circ}$ to $30^{\circ}$ \\
$\theta_{9}$ & $\mathrm{XZ}$ & Left & Ankle & $-30^{\circ}$ to $30^{\circ}$ \\
$\theta_{10}$ & $\mathrm{YZ}$ & Left & Ankle & $-20^{\circ}$ to $20^{\circ}$ \\
\hline
\end{tabular}

\section{HUMAN GAIT GENERATION FOT BIPED HUBOT-5}

Four most important variables of the humanoid robot that play an essential role in stable gait generation, including $S$ - walking step length, $H$ - Leg Lifting $[\mathrm{m}], h$ - Leg kneeling $[\mathrm{m}]$ and $n$ - Hip swinging, are clearly described in Fig. 3. In which, $d 0$ represents 
the height of the torso, $d 1$ is the distance between the 2 dof at the knee joints, $d 2$ is the length of the leg, $d 3$ is the length of the femoral and $d 4$ represents the distance between 2 hips.

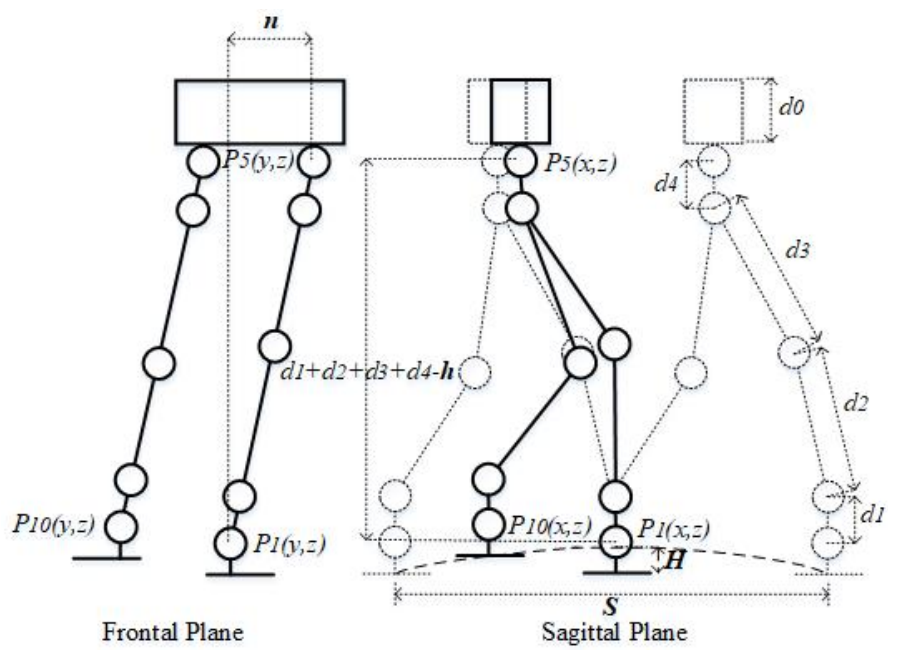

Fig. 3. Four variables influence the human walking gait of humanoid robot HUBOT-5

As described in Fig. 3, the total three trajectories of biped, including hip trajectory $P_{5}=\left[P_{5 x}, P_{5 y}, P_{5 z}\right]$ and ankle trajectory $P_{1}=\left[P_{1 x}, P_{1 y}, P_{1 z}\right]$ of the supporting leg, and ankle trajectory $P_{10}=\left[P_{10 x}, P_{10 y}, P_{10 z}\right]$ of the moving legs, will depend on 4 variables $(S$, $H, h, n)$ with respect to both of the frontal (YZ-Frontal View) and sagittal (XZ-Sagittal View) interface. The three selected trajectories $\mathbf{P}_{1}, \mathbf{P}_{5}, \mathbf{P}_{10}$ are considered as sine-time dependent, and described in Eqs. (1), (2) and (3) (see additional works at [21]).

$$
\begin{aligned}
& \left\{\begin{array}{l}
P_{1 x}(t)=\frac{S}{2} \sin \left[\frac{\pi}{T}\left(t-\frac{T}{2}\right)\right][u(t-2 T)-u(t-T)] \\
P_{1 y}(t)=w[u(t-2 T)-u(t-T)] \\
P_{1 z}(t)=H \sin \left[\pi\left(\frac{P_{1 x}(t)}{S}+0.5\right)\right][u(t-2 T)-u(t-T)],
\end{array}\right. \\
& \left\{\begin{array}{l}
P_{10 x}(t)=\frac{S}{2} \sin \left[\frac{\pi}{T}\left(t-\frac{T}{2}\right)\right][u(t)-u(t-T)] \\
P_{10 y}(t)=-w[u(t)-u(t-T)] \\
P_{10 z}(t)=H \sin \left[\pi\left(\frac{P_{10 x}(t)}{S}+0.5\right)\right][u(t)-u(t-T)]
\end{array}\right.
\end{aligned}
$$




$$
\left\{\begin{array}{c}
P_{5 x}(t)=\frac{S}{4} \sin \left(\frac{\pi}{T}\left(\tau-\frac{T}{2}\right)\right), \\
P_{5 y \_ \text {first_hal__cycle }}(t)=n \sin \left(\frac{\pi}{T} \tau\right)\left[u(\tau)-u\left(\tau-\frac{T}{2}\right)\right] \\
\quad+n \cos \left(\frac{\pi}{T}\left(\tau-\frac{T}{2}\right)\right)\left[u\left(\tau-\frac{T}{2}\right)-u(\tau-T)\right], \\
P_{5 y}(t)=P_{5 y_{-} \text {first_half_cycle }(t)[u(t)-u(t-T)]}+P_{5 y_{-} \text {irst_half_cycle }}(t)[u(t-2 T)-u(t-T)] \\
P_{6 z}(t)=\left(d_{1}+d_{2}+d_{3}+d_{4}-h\right) .
\end{array}\right.
$$

In which, $T$ represents the time to perform a step of the humanoid robot, $w$ represents the distance between 2 legs, $\tau=\left\{\begin{array}{ll}t & \text { if } 0 \leq t \leq T \\ t-T & \text { otherwise }\end{array}\right.$ and $u(t)=\left\{\begin{array}{ll}0 & \text { if } t<0 \\ 1 & \text { otherwise }\end{array}\right.$.

From Eqs. (1)-(3), both of hip and ankle trajectories of the supporting leg and ankle trajectory of the moving leg are used to generate walking gait for the humanoid robot.

Finally, the trajectories of the ten angular joints located at the 2 legs in one walking interval cycle can be defined from $P_{1}=\left[P_{1 x}, P_{1 y}, P_{1 z}\right], P_{5}=\left[P_{5 x}, P_{5 y}, P_{5 z}\right]$ and $P_{10}=$ $\left[P_{10 x}, P_{10 y}, P_{10 z}\right]$, and based on the biped inverse kinematics. The biped inverse kinematics can be conventionally solved by calculus or numerical methods. However, in this section, the geometric method based on the HUBOT-5 robot rotary joint will be shown, as described in the Eq. (4).

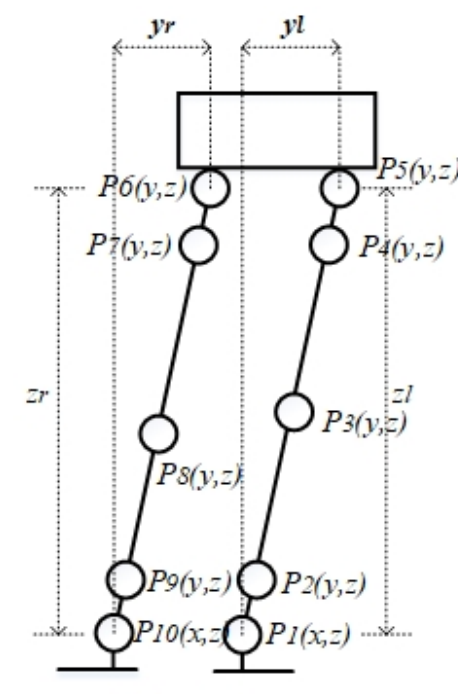

Frontal Plane

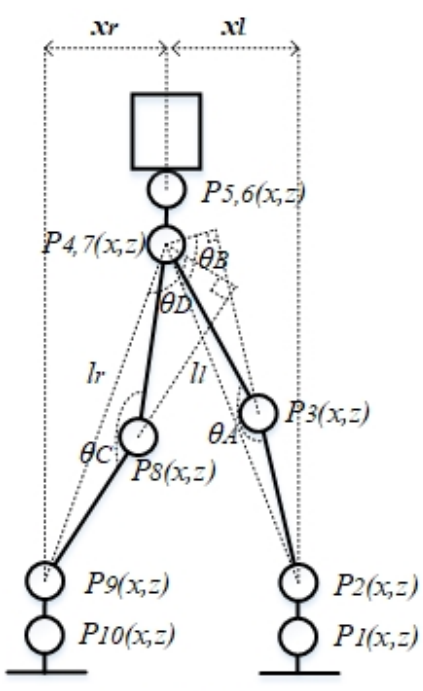

Sagittal Plane

Fig. 4. Variables defined in formula (4) 
In which, $y_{l}(t), z_{l}(t), y_{r}(t), z_{r}(t), \theta_{A}(t), \theta_{B}(t), \theta_{C}(t), \theta_{D}(t), x_{l}(t), x_{r}(t), l_{l}(t)$, $l_{r}(t)$ at specified time $t$, are defined as in Fig. 4 and Eq. (5); represents the distance between $\mathbf{P}_{2}$ and $\mathbf{P}_{4}$, represents the distance between $\mathbf{P}_{9}$ and $\mathbf{P}_{7}$.

$$
\left\{\begin{array}{l}
\theta_{1}(t)=\arctan \left(\frac{y_{l}(t)}{z_{l}(t)}\right), \theta_{5}(t)=-\theta_{1}(t), \\
\theta_{10}(t)=\arctan \left(\frac{y_{r}(t)}{z_{r}(t)}\right), \theta_{6}(t)=-\theta_{10}(t), \\
\theta_{3}(t)=\pi-\theta_{A}(t), \theta_{8}(t)=\pi-\theta_{C}(t), \\
\theta_{4}(t)=\frac{\pi}{2}-\theta_{A}(t)+\theta_{B}(t)-\arcsin \left(\frac{x_{l}(t)}{l_{l}(t)}\right), \\
\theta_{7}(t)=\frac{\pi}{2}-\theta_{C}(t)+\theta_{D}(t)-\arcsin \left(\frac{x_{r}(t)}{l_{r}(t)}\right), \\
\theta_{2}(t)=\theta_{3}(t)-\theta_{4}(t), \theta_{11}(t)=\theta_{9}(t)-\theta_{7}(t), \\
x_{l}=\sqrt{\left(P_{4 x}-P_{2 x}\right)^{2}+\left(P_{4 y}-P_{2 y}\right)^{2}+\left(P_{4 z}-P_{2 z}\right)^{2},} \\
x_{r}=P_{6 x}-P_{10 x}, y_{r}=P_{6 y}-P_{10 y}, \quad z_{r}=P_{6 z}-P_{10 z}, \\
l_{r}=\sqrt{\left(P_{7 x}-P_{9 x}\right)^{2}+\left(P_{7 y}-P_{9 y}\right)^{2}+\left(P_{7 z}-P_{9 z}\right)^{2},} \\
\theta_{A}=\arccos \left(\frac{d_{2}^{2}+d_{3}^{2}-l_{l}^{2}}{2 d_{2} d_{3}}\right), \theta_{B}=\arccos \left(\frac{d_{3} \sin \left(\theta_{A}\right)}{l_{l}}\right), \\
\theta_{C}=\arccos \left(\frac{d_{2}^{2}+d_{3}^{2}-l_{r}^{2}}{2 d_{2} d_{3}}\right), \theta_{D}=\arccos \left(\frac{d_{3} \sin \left(\theta_{C}\right)}{l_{l}}\right) .
\end{array}\right.
$$

In which $d_{1}, d_{2}, d_{3}$ and $d_{4}$ are illustrated in Fig. 3. The coordination $P_{6}(x, y, z)$ is calculated based on $P_{5}(x, y, z)$, and the coordination of $\left[P_{2}(x, y, z), P_{4}(x, y, z), P_{7}(x, y, z)\right.$, $\left.P_{9}(x, y, z)\right]$ is calculated based on $\left[P_{1}(x, y, z), P_{5}(x, y, z), P_{6}(x, y, z), P_{10}(x, y, z)\right]$ and the rotrary angle $\left[\theta_{1}, \theta_{5}, \theta_{6}, \theta_{10}\right]$. Eqs. (6) below are used to determine, $P_{2}, P_{4}, P_{6}, P_{7}, P_{9}$.

$$
\left\{\begin{array}{l}
P_{2 x}=P_{1 x}, \quad P_{2 z}=d_{1} \cos \left(\theta_{1}\right), \quad P_{2 y}=P_{2 z} \sin \left(\theta_{1}\right), \\
P_{4 x}=P_{5 x}, \quad P_{4 z}=P_{5 z}-d_{4} \cos \left(\theta_{1}\right), \quad P_{4 y}=P_{5 y}-\left(P_{5 z}-P_{4 z}\right) \sin \left(\theta_{1}\right), \\
P_{6 x}=P_{5 x}, \quad P_{6 y}=P_{5 y}-w, \quad P_{6 z}=P_{5 z}, \\
P_{7 x}=P_{6 x}, \quad P_{7 z}=P_{6 z}-d_{4} \cos \left(\theta_{10}\right), \quad P_{7 y}=P_{6 y}-\left(P_{6 z}-P_{7 z}\right) \sin \left(\theta_{10}\right) \\
P_{9 x}=P_{10 x}, \quad P_{9 z}=P_{10 z}+d_{4} \cos \left(\theta_{10}\right), \quad P_{9 y}=P_{10 y}+\left(P_{9 z}-P_{10 z}\right) \sin \left(\theta_{10}\right) .
\end{array}\right.
$$

In summary, using Eqs. (4)-(6), the ten trajectories of the rotary angles located at the 2 legs of biped HUBOT-5 in one interval walking cycle are computed to accurately and efficiently control the biped walking gait.

Thus the set of four parameters $H, h, s$ and $n$ need to be optimally selected so that the resulted ZMP parameter ensures that the biped robot can walk steadily with the preset foot-lift value. In this paper the CFO-Central Force Optimization algorithm is used to satisfactorily solve this task. 


\section{GAIT PARAMETRIC OPTIMIZATION USING CFO}

\subsection{CFO algorithm}

The CFO was first introduced in 2007 by R. A. Formato [22]. The algorithm is a somewhat new optimization technique which is nature-based, multidimensional, metaheuristic, and based heavily on the gravitational kinematics concept, for example, object fly through space. Unlike other evolutionary algorithms (GA, PSO), CFO does not contain random characteristic, and that makes the $\mathrm{CFO}$ deterministic.

Concretely the proposed CFO algorithm consists of following steps:

(1) Calculate initial posision of probe, evaluate their value and their accelerations, then compute their mass.

(2) Based on the evaluated acceleration, find new position of the probes.

(3) Check if each probe mass and position is located inside the decision space, or whether is it converged.

(4) Update the fitness value with new position.

(5) Update acceleration.

(6) Loop until stopping criterion is satisfied.

It is also important to notice the followed CFO notable parameters:

- Alpha coefficient represents the influence of probes to each other, using the difference of mass. The lower alpha is the less effect that probe with approximate mass will influence each other.

- Beta, as distance extended, determines the remaining influence of probes to each other in space. The lower beta is the more effect one probe can have to others as great distance.

- Gamma, simply determines accelerations, the higher gamma is, the faster the probe accelerate. This can be good when the user want to quickly find the optimal solution. However, this may have a bad effect on the convergence characteristics.

\subsection{Specification of the objective function}

The objective function must be defined to evaluate gait parameters of the humanoid robot. The goal of the HUBOT-5 biped robot is to achieve a stable gait with preset footlifting value. For this purpose, the ZMP point projection on the foot area based on the ZMP principle will be used.

When the feet touch the ground, the area of the supporting foot is the area between the two feet of the human robot, and when one foot touches the ground, the foot area is the surface of the foot touching the ground. The supporting foot area in the two cases is illustrated in Fig. 5.

The zero moment point $\mathrm{ZMP}$ is a point in the plane where the total external torque applied to the humanoid robot at this point is zero. Then if the ZMP is within the area of the supporting leg, the robot does not fall. The calculation of the ZMP trajectory of biped robots in walking is shown in Section 4.3. 


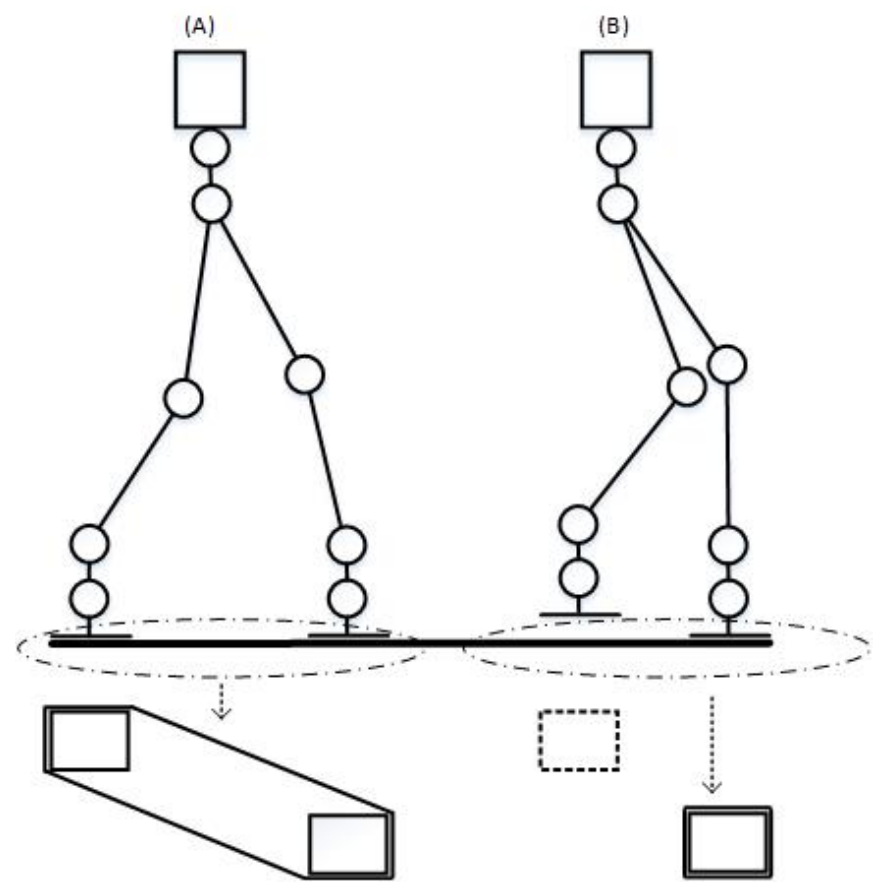

Fig. 5. Area of the supporting foot in cases: (a) 2 foot on the ground, (b) 1 foot on the ground

The sum of the squared distance from the ZMP to the center of the supporting foot in 1 step walking of biped HUBOT-5, see Eq. (7), represents the first objective function.

$$
f_{1}=\int_{0}^{T} \sqrt{x_{Z M P}^{2}+y_{Z M P}^{2}} \mathrm{~d} t
$$

with $T$ denotes stepping cycle and $\left(x_{Z M P}, y_{Z M P}\right)$ denotes the coordination of ZMP in the process of the biped robot performs the step. The smaller $f_{1}$ is the more stable the gait will become.

Additionally, for the humanoid robot to follow the pre-set foot-lifting height value $-H_{r e f}$, the difference between the magnitude of the foot-lift parameter - and the foot-lift preset value $-H_{r e f}$ (see Eq. (8)) represents the second objective function. Eq. (8) means that the smaller $f_{2}$ is the more strictly the lifting magnitude $H$ can follow $H_{r e f}$.

$$
f_{2}=\left|H_{\text {ref }}-H\right| \text {. }
$$

Thus, in order for biped HUBOT-5 to obtain a steady gait with the foot-lift set up in advance, we find the minimum value of the two objective functions $f_{1}$ and $f_{2}$, or similarly to find the minimum of the function $f$ as

$$
f=\lambda\left(\int_{0}^{T} \sqrt{x_{Z M P}^{2}+y_{Z M P}^{2}} \mathrm{~d} t\right)+(1-\lambda)\left|H_{r e f}-H\right| .
$$


In which, $\lambda(0<\lambda \leq 1)$ is optimally selected as to prioritize between the walking stability ( $\lambda$ increase) and the variance with the desired foot-lifting magnitude ( $\lambda$ decreased).

\subsection{ZMP trajectory calculation}

The ZMP trajectory resulted from calculated ZMP point which is computed using Eq. (10) [6]

$$
\left\{\begin{array}{l}
x_{Z M P}=\frac{\sum_{i=1}^{n} m_{i}\left(\ddot{z}_{i}+g\right) x_{i}-\sum_{i=1}^{n} m_{i} \ddot{x}_{i} z_{i}-\sum_{i=1}^{n} I_{i y} \ddot{\Omega}_{i y}}{\sum_{i=1}^{n} m_{i}\left(\ddot{z}_{i}+g\right)}, \\
y_{Z M P}=\frac{\sum_{i=1}^{n} m_{i}\left(\ddot{z}_{i}+g\right) y_{i}-\sum_{i=1}^{n} m_{i} \ddot{y}_{i} z_{i}-\sum_{i=1}^{n} I_{i x} \ddot{\Omega}_{i x}}{\sum_{i=1}^{n} m_{i}\left(\ddot{z}_{i}+g\right)} .
\end{array}\right.
$$

In which, $m_{i}$ represents the weight of the $i^{\text {th }}$ stage and $\left(x_{i}, y_{i}, z_{i}\right)$ is the coordinates of the center of the $i^{\text {th }}$ stage in the Descartes coordinate system, $I_{i x}$ and $I_{i y}$ denote properties of the inertia momentum, $\ddot{\Omega}_{i x}$ and $\ddot{\Omega}_{i y}$ are the acceleration properties of rotary angle around $x$ and $y$ at the center $i^{\text {th }}$ joint, $g$ denotes gravitational acceleration, $\left(x_{Z M P}, y_{Z M P}\right)$ represent the ZMP coordinations.

For biped HUBOT-5, the weight of the links is concentrated on the mass of the joints, so the center of the joint is considered to be located at the end of the joint. Thus, the inertial moment term of Eq. (10) is considered zero, then the Equation for ZMP is calculated as (11)

$$
\left\{\begin{aligned}
x_{\mathrm{ZMP}} & =\frac{\sum_{i=1}^{n} m_{i}\left(\ddot{z}_{i}+g\right) x_{i}-\sum_{i=1}^{n} m_{i} \ddot{x}_{i} z_{i}}{\sum_{i=1}^{n} m_{i}\left(\ddot{z}_{i}+g\right)} \\
y_{\mathrm{ZMP}} & =\frac{\sum_{i=1}^{n} m_{i}\left(\ddot{z}_{i}+g\right) y_{i}-\sum_{i=1}^{n} m_{i} \ddot{y}_{i} z_{i}}{\sum_{i=1}^{n} m_{i}\left(\ddot{z}_{i}+g\right)}
\end{aligned}\right.
$$

In (11), the mass distribution $m_{i}$ and coordinates $\left(x_{i}, y_{i}, z_{i}\right)$ of the stages are defined in Fig. 6.

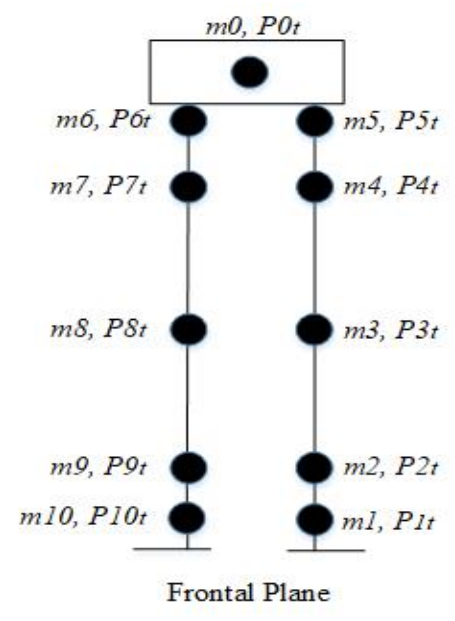

Fig. 6. The mass distribution and coordinates of the stages 
The coordinates $P_{i t}(x, y, z)$ of the joint are determined from the 10 angles of rotation at one time in one step with the original coordinatation at the center of the suppoting foot by means of the geometric Equation (12)

$$
\left\{\begin{array}{l}
P_{1 x t}(t)=0, P_{1 y t}(t)=0, P_{1 z t}(t)=0, \\
P_{2 x t}(t)=P_{1 x t}, P_{2 z t}(t)=d_{1} \cos \left[\theta_{1}(t)\right], P_{2 y t}(t)=P_{2 z t}(t) \sin \left[\theta_{1}(t)\right], \\
P_{3 x t}(t)=d_{2} \sin \left[\theta_{2}(t)\right], P_{3 y t}(t)=P_{3 z t}(t) \sin \left[\theta_{1}(t)\right], \\
P_{3 z t}(t)=P_{2 z t}(t)+d_{2} \cos \left[\theta_{2}(t)\right] \cos \left[\theta_{1}(t)\right], \\
P_{4 x t}(t)=P_{3 x t}(t)+d_{3} \sin \left[\theta_{2}(t)-\theta_{3}(t)\right], \\
P_{4 y t}(t)=P_{4 z t}(t) \sin \left[\theta_{1}(t)\right], \\
P_{4 z t}(t)=P_{3 z t}(t)+d_{3} \cos \left[\theta_{2}(t)-\theta_{3}(t)\right] \cos \left[\theta_{1}(t)\right], \\
P_{5 x t}(t)=P_{4 x t}(t)+d_{4} \sin \left[\theta_{2}(t)-\theta_{3}(t)+\theta_{5}(t)\right], \\
P_{5 y t}(t)=P_{5 z t}(t) \sin \left[\theta_{1}(t)\right], \\
P_{5 z t}(t)=P_{4 z t}(t)+d_{4} \cos \left[\theta_{2}(t)-\theta_{3}(t)+\theta_{5}(t)\right] \cos \left[\theta_{1}(t)\right], \\
P_{6 x t}(t)=P_{5 x t}(t), P_{6 y t}(t)=P_{5 y t}(t)-w, P_{6 z t}(t)=P_{5 z t}(t), \\
P_{7 x t}(t)=P_{6 x t}(t), P_{7 z t}(t)=P_{6 z t}(t)-d_{4} \cos \left[\theta_{6}(t)\right], \\
P_{7 y t}(t)=P_{6 y t}(t)-\left[P_{6 z t}(t)-P_{7 z t}(t)\right] \sin \left[\theta_{6}(t)\right], \\
P_{8 x t}(t)=P_{7 x t}(t)+d_{3} \sin \left[\theta_{7}(t)\right], \\
P_{8 z t}(t)=P_{7 z t}(t)-d_{3} \cos \left[\theta_{7}(t)\right] \cos \left[\theta_{6}(t)\right], \\
P_{8 y t}(t)=P_{7 y t}(t)-\left[P_{7 z t}(t)-P_{8 z t}(t)\right] \sin \left[\theta_{6}(t)\right], \\
P_{9 x t}(t)=P_{8 x t}(t)+d_{2} \sin \left[\theta_{7}(t)-\theta_{8}(t)\right], \\
P_{9 z t}(t)=P_{8 z t}(t)-d_{2} \cos \left[\theta_{7}(t)-\theta_{8}(t)\right] \cos \left[\theta_{6}(t)\right], \\
P_{9 y t}(t)=P_{7 y t}(t)-\left[P_{7 z t}(t)-P_{9 z t}(t)\right] \sin \left[\theta_{6}(t)\right] \\
P_{10 x t}(t)=P_{11 x t}(t)+d_{1} \sin \left[\theta_{7}(t)-\theta_{8}(t)+\theta_{1}(t)\right], \\
P_{10 z t}(t)=P_{9 z t}(t)-d_{1} \cos \left[\theta_{7}(t)-\theta_{8}(t)+\theta_{9}(t)\right] \cos \left[\theta_{6}(t)\right], \\
P_{10 y t}(t)=P_{7 y t}(t)-\left[P_{7 z t}(t)-P_{10 z t}(t)\right] \sin \left[\theta_{6}(t)\right], \\
P_{0 x t}(t)=\frac{P_{5 x t}(t)+P_{6 x t}(t)}{2}, P_{1 y t}(t)=\frac{P_{5 y t}(t)+P_{6 y t}(t)}{2} \\
P_{0 z t}(t)=P_{5 z t}(t)+\frac{d_{0}}{2} . \\
\end{array}\right.
$$

In which, $d_{0}, d_{1}, d_{2}, d_{3}$ and $d_{4}$ are illustrated in Fig. 3.

Finally, the flow chart used to calculate the ZMP trajectory based on the optimal set of four gait parameters of the humanoid robot is illustrated in Fig. 7.

\section{SIMULATION AND PRACTICAL EXPERIMENT RESULTS}

The simulated and experimental results are fully tested on the small-sized HUBOT5 biped robot. The physical parameters of the HUBOT-5 biped robot are presented in Tab. 2.

In order to find the most appropriate value for the coefficients $\lambda$ of the objective function in Eq. (9), based on CFO algorithm, it optimally selects $\lambda=0.4$ which permits the HUBOT-5 biped robot attaining a steady gait with an adjustable foot-lift value, and this $\lambda$ value will be used thorough the comparative testing process using GA, PSO, MDE and CFO. 


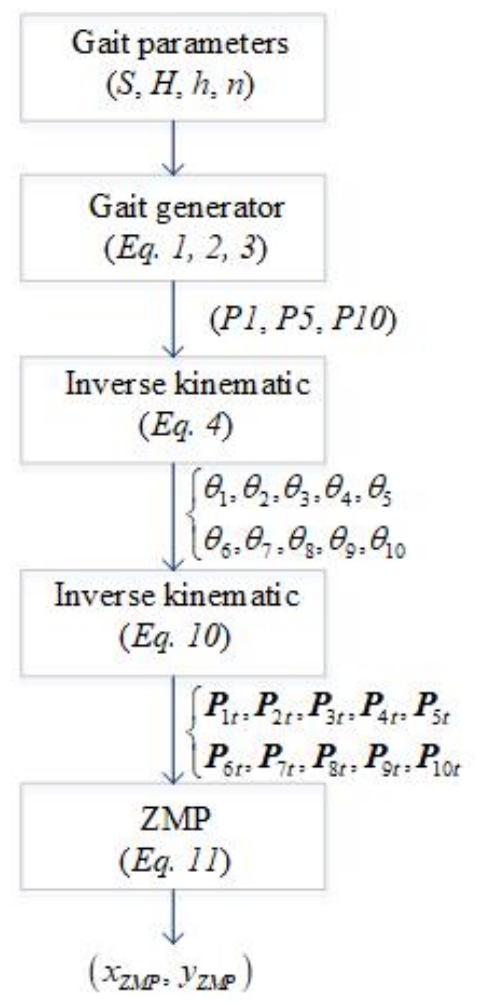

Fig. 7. ZMP calculation flow chart

Table 2. Physical parameters of biped HUBOT-5

\begin{tabular}{|c|c|}
\hline Parameters & Value \\
\hline$d_{0}$ & $6.000 \mathrm{~cm}$ \\
\hline$d_{1}$ & $4.254 \mathrm{~cm}$ \\
\hline$d_{2}$ & $9.109 \mathrm{~cm}$ \\
\hline$d_{3}$ & $8.063 \mathrm{~cm}$ \\
\hline$d_{4}$ & $9.345 \mathrm{~cm}$ \\
\hline$w$ & $8.640 \mathrm{~cm}$ \\
\hline $\begin{array}{l}m_{i} \\
i=0 \ldots 10\end{array}$ & 60 gam \\
\hline
\end{tabular}

Table 3. Bounds for 4 walking gait paramteres of humanoid HUBOT-5

\begin{tabular}{lcc}
\hline Parameters & Lower Bound & Upper Bound \\
\hline S-Step length & $1.5 \mathrm{~cm}$ & $25 \mathrm{~cm}$ \\
$H$-foot lifting & $0.1 \mathrm{~cm}$ & $10 \mathrm{~cm}$ \\
$h$-kneeling & $0.1 \mathrm{~cm}$ & $1.5 \mathrm{~cm}$ \\
n-hip swinging & $0.1 \mathrm{~cm}$ & $10 \mathrm{~cm}$ \\
\hline
\end{tabular}

For optimal test based on GA, PSO, MDE and CFO optimization algorithms, the gait parameters derived from the biped HUBOT-5 are limited as given in Tab. 3.

The mathematical properties of GA, PSO, MDE, and CFO optimization algorithms are meta-heuristic algorithms, so each algorithm will perform 10 different training times, with each training will repeat 500 times $(N=500)$ using the same population size 
$(\mathrm{NP}=32)$ and the same number of variables $(n=4)$. Tab. 4 eventually presents the GA, PSO, MDE and CFO selected parametric values.

Table 4. Parameters of GA, PSO, MDE and CFO algorithm

\begin{tabular}{clc}
\hline Method & \multicolumn{1}{c}{ Paramters } & Value \\
\hline GA & Mutation $(\mathrm{F})$ & 0.4 \\
& Crossover Probability $(\mathrm{CR})$ & 0.9 \\
PSO & Accelaration factor $\left(\mathrm{C}_{1}\right)$ & 2.0 \\
& Accelaration factor $\left(\mathrm{C}_{2}\right)$ & 2.0 \\
& Inertia Weight $(w)$ & {$[0.4 ; 0.9]$} \\
MDE & Mutation value $(\mathrm{F})$ & Random $[0.4 ; 1.0]$ \\
& Crossover Probability $(\mathrm{CR})$ & Random $[0.7 ; 1.0]$ \\
& Alpha & 0.25 \\
& Beta & 0.35 \\
& Gamma & 0.95 \\
& Frep & 0.5 \\
& deltaFrep & 0.05 \\
\hline
\end{tabular}

Specify the foot-lifting height of HUBOT-5 being $H_{\text {ref }}=20 \mathrm{~mm}$. Fig. 8 illustrates the mean value of the target function after 10 runs of each algorithm (GA: green, PSO: blue, MDE: red, CFO: reddish purple).

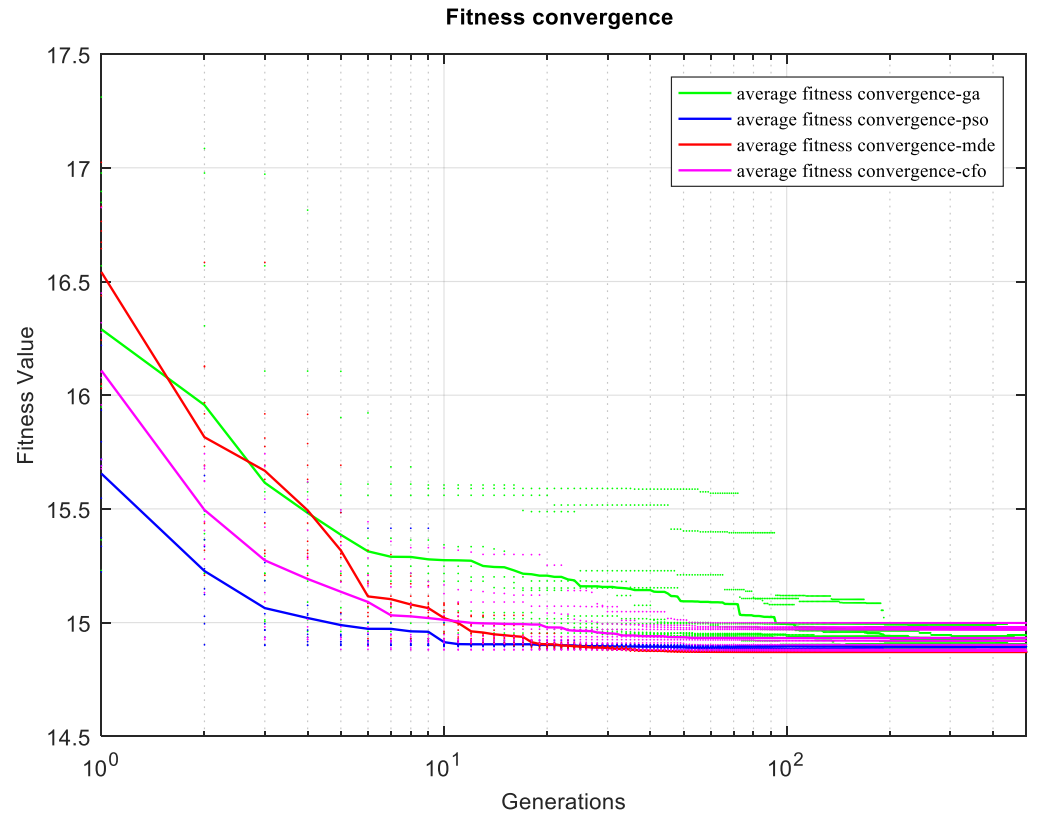

Fig. 8. Mean value of $f$ 
Derived from Tab. 5, the optimum set of parameters for the biped HUBOT-5 conformed to the objective of 10 runs per GA, PSO, MDE and CFO algorithms is shown in Tab. 5. Fig. 9 shows resulted comparative ZMP and COM trajectories when HUBOT-5 steps along with a stepping cycle $(T=2 \mathrm{~s})$ with respect to the configurations based on GA, PSO, MDE and CFO algorithms, respectively.

Table 5. Resulted parametric set for four comparative algorithms

\begin{tabular}{cccccc}
\hline \multicolumn{5}{c}{$H_{\text {ref }}=2 \mathrm{~cm}$} \\
\cline { 2 - 4 } Agorithms & \multicolumn{3}{c}{ Walking Gait Parameters value } & \multirow{2}{*}{$\begin{array}{c}\text { Best firness } \\
\text { value } f(\mathrm{~cm})\end{array}$} \\
\cline { 2 - 4 } & $S(\mathrm{~cm})$ & $H(\mathrm{~cm})$ & $h(\mathrm{~cm})$ & $n(\mathrm{~cm})$ & valu. \\
GA & 15 & 1.99 & 0.82 & 6.99 & 14.87 \\
PSO & 15 & 2.00 & 0.8 & 6.89 & 14.87 \\
MDE & 15 & 2.00 & 0.8 & 6.89 & 14.87 \\
CFO & 15 & 2.00 & 0.89 & 6.99 & 14.87 \\
\hline
\end{tabular}

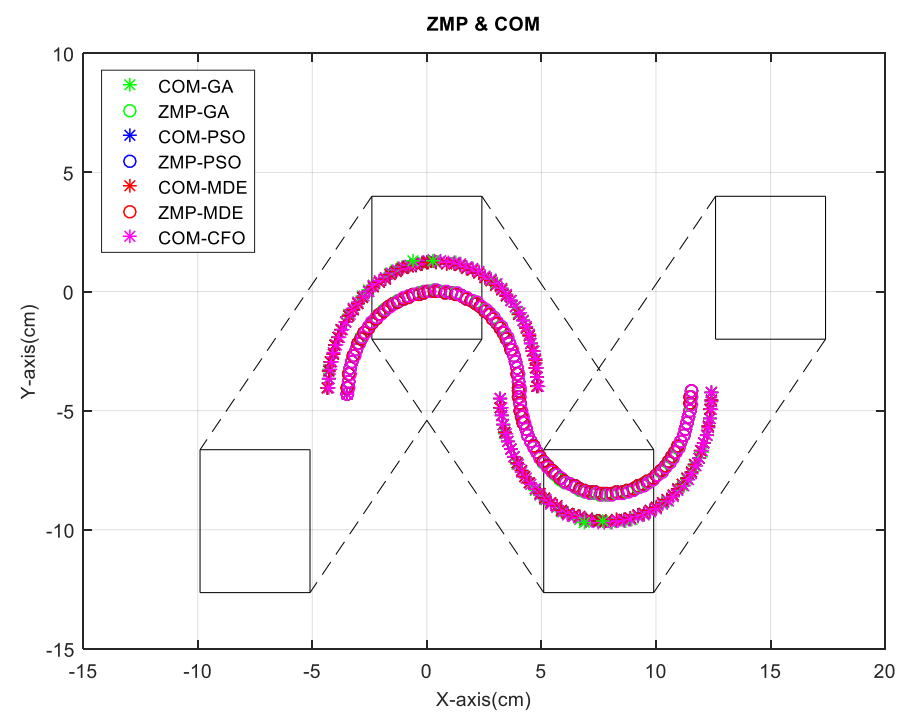

Fig. 9. Resulted comparative ZMP and COM survey

The optimal set of parameters for four comparative algorithms presented in Tab. 6 shows that the target is reached with respect to the preset foot-lift value. The ZMP and COM trajectories corresponding to each of the four comparative algorithms presented in Fig. 9 shows that they are always within the footprint and this means that biped HUBOT5 are achieving steady-state stable and robust walking.

Based on the results described in Fig. 8, it is important to notice that the MDE algorithm searches for an optimal solution with an average value of 14.8706499 after about 119 
generations, while the PSO algorithm is approximately 211 generations after the search, finding an optimal solution obtained an average value of 14.88034529 , while the GA algorithm must need around 470 generations to find the optimal solution with an average value of 14.9039. The proposed CFO algorithm finds an optimal solution of 14.93174983 after an average of 89 generations. These results show that the $\mathrm{CFO}$ algorithm outperforms GA, PSO, and MDE algorithms in terms of convergence speed.

Tab. 6 demonstrates the optimized value of the walking gait parameters to ensure the biped HUBOT-5 to walk steadily with both cases corresponding to differnet preset foot-lift magnitude. $\left(H_{r e f}=2 \mathrm{~cm}\right.$ and $\left.H_{r e f}=4 \mathrm{~cm}\right)$ using CFO.

Table 6. The optimized value of the walking gait parameters

\begin{tabular}{ccccc}
\hline \multirow{2}{*}{$H_{\text {ref }}(\mathrm{cm})$} & \multicolumn{4}{c}{ CFO optimization results } \\
\cline { 2 - 5 } & $S(\mathrm{~cm})$ & $H(\mathrm{~cm})$ & $h(\mathrm{~cm})$ & $n(\mathrm{~cm})$ \\
\hline 2.0 & 15 & 2.0 & 0.89 & 6.99 \\
4.0 & 15 & 4.0 & 1.09 & 7.12 \\
\hline
\end{tabular}

Fig. 10 illustrates the 2D gait in the X-Z plane of the HUBOT-5, corresponding to two cases with different foot lifts. Tab. 6 and Fig. 11 show that the biped HUBOT- 5 has a pickup lift in the set value.
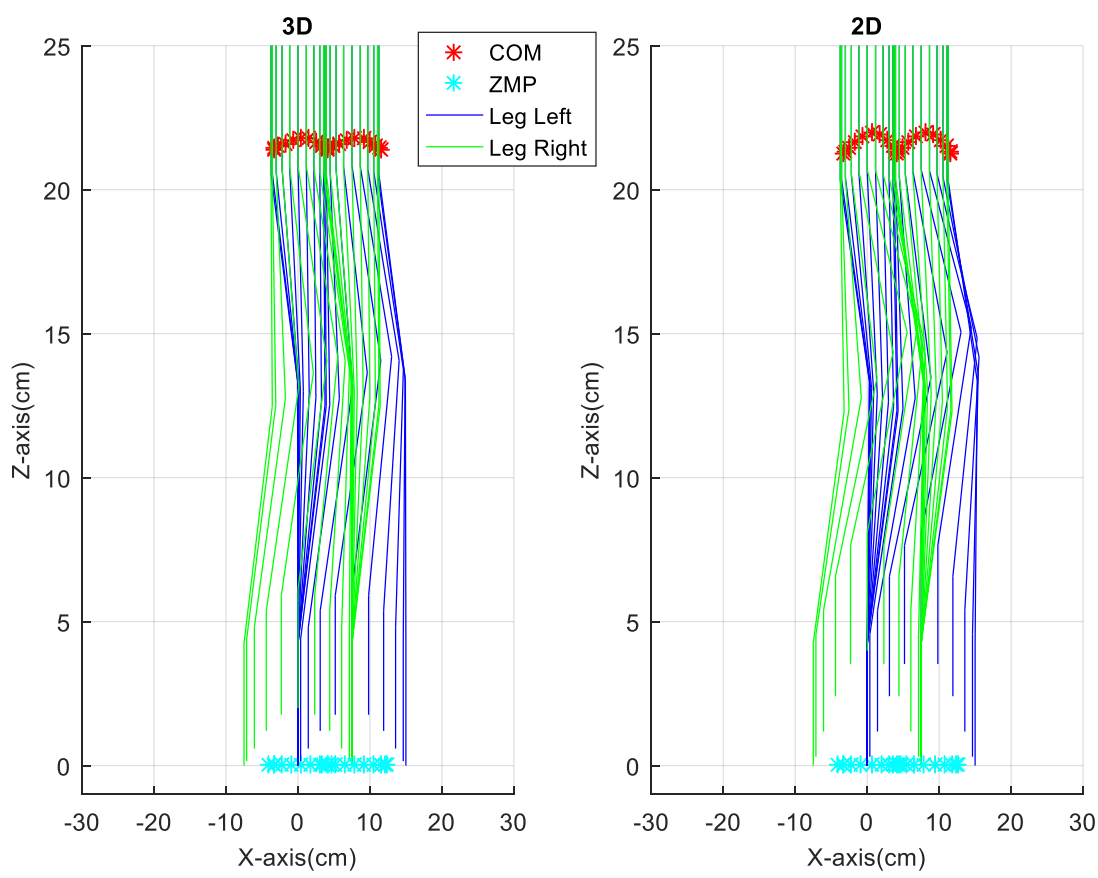

Fig. 10. Simulated 2D gait result of biped HUBOT-5 with different foot-lifting amplitudes 
Fig. 11 illustrates the resulted ZMP point trajectory and the projection of COM trajectory for two different foot-lifting amplitudes. This shows that the ZMP point is always in the supporting foot area and then it ensures that the HUBOT- 5 biped robot keeps stable walking.
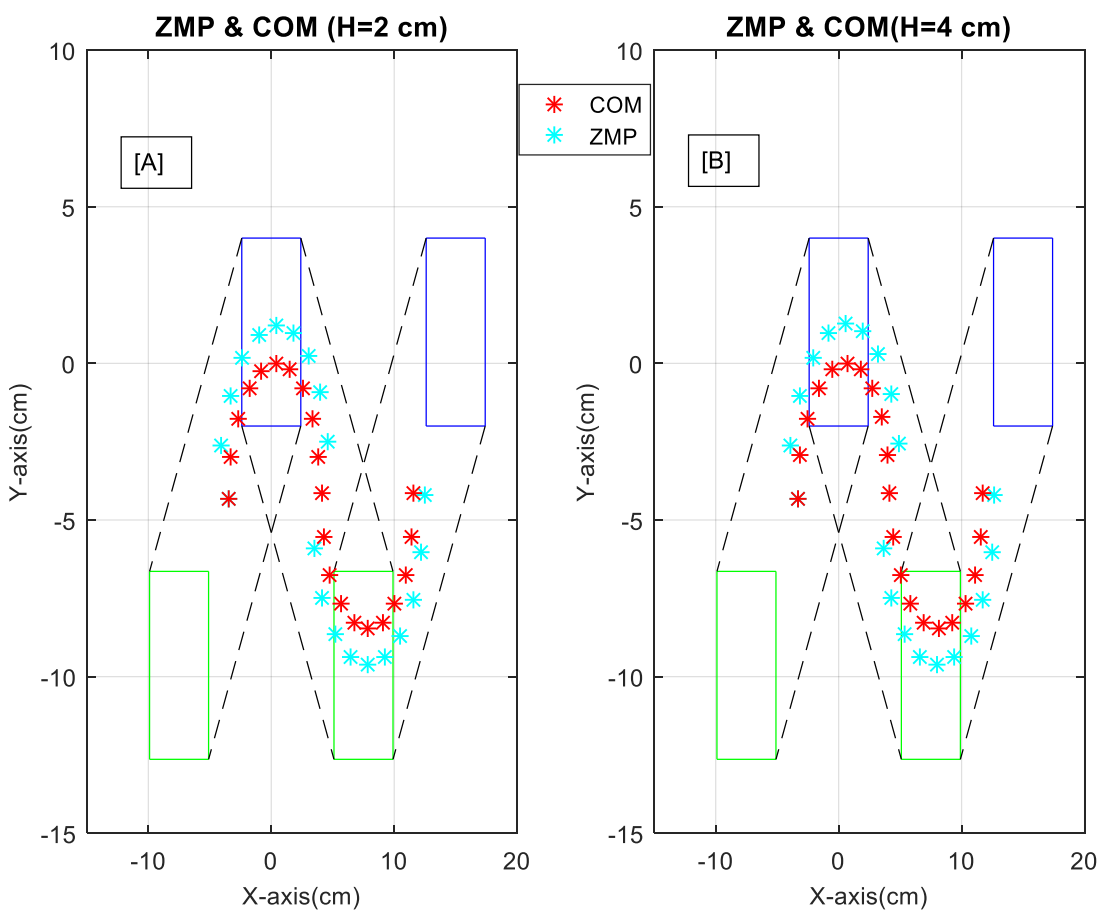

Fig. 11. Resulted ZMP and COM trajectories

Using ten rotary angular values to control the biped HUBOT-5, it performs two corresponding steps with two different foot-lift values. Fig. 12 illustrates the photos of the HUBOT-5 biped robot in performing a stable and steady walking step with respect to the foot-lift value $H_{\text {ref }}=4 \mathrm{~cm}$.

Fig. 13 demonstrates the ten rotary angular trajectorial errors in one stepping cycle of the two legs of experimental biped HUBOT- $5\left(\theta_{1}, \theta_{2}, \theta_{3}, \theta_{4}, \theta_{5}, \theta_{6}, \theta_{7}, \theta_{8}, \theta_{9}, \theta_{10}\right)$ during walking with optimally resulted sets of gait parameters in term of pre-set foot-lifting value $H_{\text {ref }}=2 \mathrm{~cm}$ (tabulated in Tab. 6).

The simulation and experiment results abovementioned convincingly ensure that the HUBOT-5 biped robot steadily walks without falling apart and keeping pace with desired foot-lift amplitude. Hence the proposed algorithm with gait parameters optimized by $\mathrm{CFO}$ algorithm is quite feasible. 


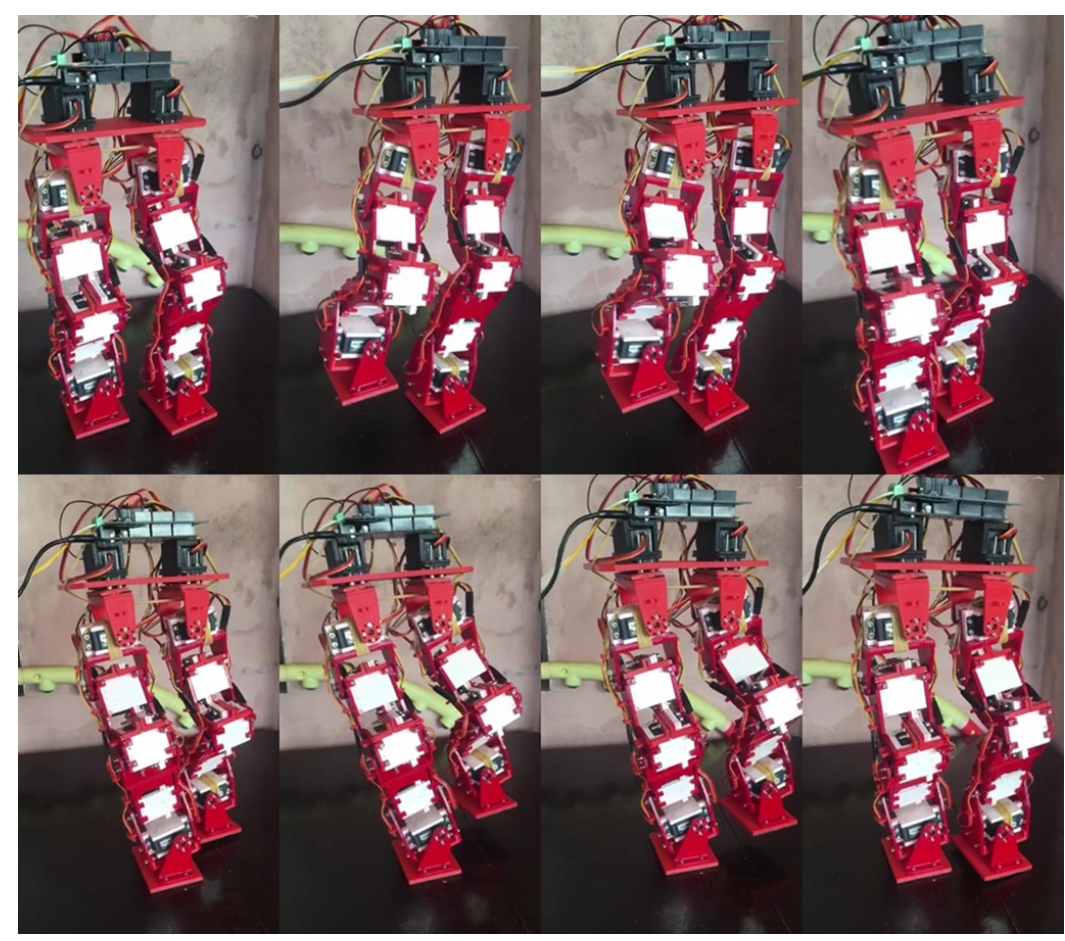

Fig. 12. Photos of experiment biped HUBOT-5 performing stable gait with foot-lifting amplitude of $H_{r e f}=4 \mathrm{~cm}$
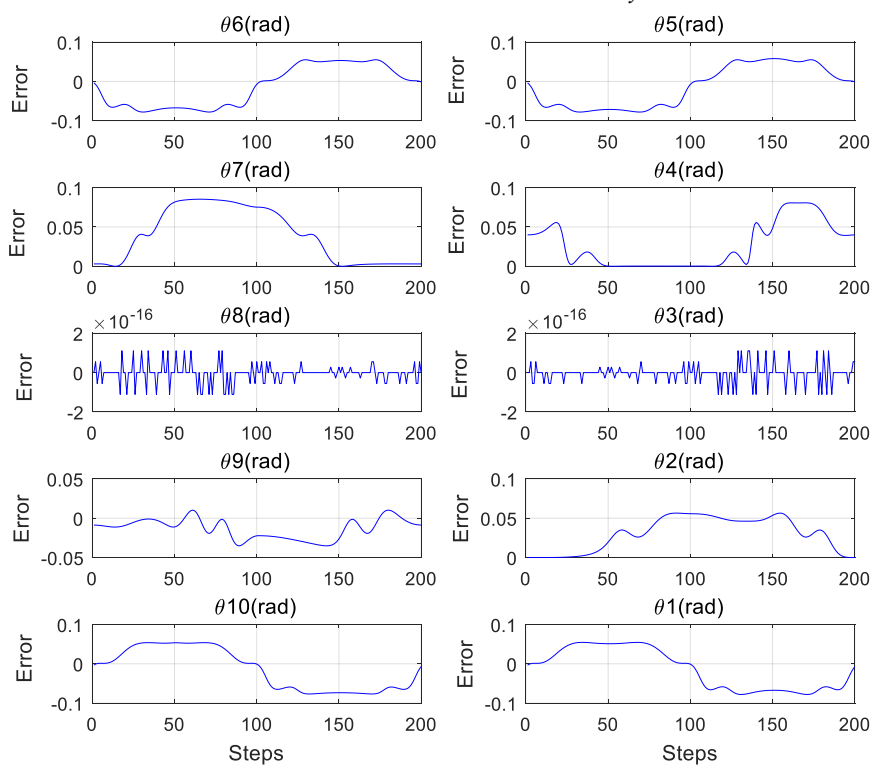

Fig. 13. The ten rotary angular trajectorial errors of two legs of experiment biped HUBOT-5 $\left(H_{r e f}=2 \mathrm{~cm}\right)$ 


\section{CONCLUSION}

In this paper, we propose a new study using the CFO algorithm as to optimize the parameters of walking pattern generation for biped robots that permits stable and robust stepping with pre-set foot-lifting magnitude. Completed experiments are determined based on novel gait generator of the two-foot trajectory, hip trajectory and the inverse kinematics collected from the human robot walking. The stable gait generation of biped robot is determined based on four key biped walking parameters: walking step length, leg lifting, leg kneeling, and hip swinging. The CFO optimization algorithm is applied to find the best solution for human robotic gait parameters so that the ZMP distance to the center of the supporting foot attains the smallest with respect to the preset footlifting value. The simulated and experimental results of proposed algorithm applied on the small-sized biped HUBOT-5 robot demonstrate the performance of novel algorithm allowing the biped robot to move steadily with an effectively reduced training time.

\section{ACKNOWLEDGMENT}

This work is fully supported by National Foundation of Science and Technology Development (NAFOSTED) under Grant 107.01-2018.10.

\section{REFERENCES}

[1] W. T. Miller. Real-time neural network control of a biped walking robot. IEEE Control Systems, 14, (1), (1994), pp. 41-48. https://doi.org/10.1109/37.257893.

[2] C.-L. Shih. Ascending and descending stairs for a biped robot. IEEE Transactions on Systems, Man, and Cybernetics-Part A: Systems and Humans, 29, (3), (1999), pp. 255-268. https://doi.org/10.1109/3468.759271.

[3] S. Kajita, F. Kanehiro, K. Kaneko, K. Fujiwara, K. Harada, K. Yokoi, and H. Hirukawa. Biped walking pattern generation by using preview control of zero-moment point. In Proceedings of the IEEE International Conference on Robotics and Automation, Taipei, Taiwan, (2003). pp. 14-19.

[4] Y.-F. Ho, T.-H. S. Li, P.-H. Kuo, and Y.-T. Ye. Parameterized gait pattern generator based on linear inverted pendulum model with natural ZMP references. The Knowledge Engineering Review, 32, (2017). https://doi.org/10.1017/S0269888916000138.

[5] J. Mrozowski, J. Awrejcewicz, and P. Bamberski. Analysis of stability of the human gait. Journal of Theoretical and Applied Mechanics, 45, (1), (2007), pp. 91-98.

[6] Q. Huang, K. Yokoi, S. Kajita, K. Kaneko, H. Arai, N. Koyachi, and K. Tanie. Planning walking patterns for a biped robot. IEEE Transactions on Robotics and Automation, 17, (3), (2001), pp. 280-289. https://doi.org/10.1109/70.938385.

[7] V.-H. Dau, C.-M. Chew, and A.-N. Poo. Optimal trajectory generation for bipedal robots. In Proceedings IEEE-RAS International Conference on Humanoid Robot, Pittsburgh, PA, USA, (2007). IEEE, pp. 603-608.

[8] G. Dip, V. Prahlad, and P. D. Kien. Genetic algorithm-based optimal bipedal walking gait synthesis considering tradeoff between stability margin and speed. Robotica, 27, (3), (2009), pp. 355-365. https://doi.org/10.1017/S026357470800475X.

[9] M. R. Maximo, E. L. Colombini, and C. H. C. Ribeiro. Stable and fast model-free walk with arms movement for humanoid robots. International Journal of Advanced Robotic Systems, 14, (3), (2017). https://doi.org/10.1177/1729881416675135. 
[10] R. Khusainov, A. Klimchik, and E. Magid. Kinematic and dynamic approaches in gait optimization for humanoid robot locomotion. In Informatics in Control, Automation and Robotics. Springer, (2018), pp. 293-320.

[11] T. T. Huan and H. P. H. Anh. Novel stable walking for humanoid robot using particle swarm optimization algorithm. Journal of Advances in Intelligent Systems Research, 123, (2015), pp. 322-325.

[12] T. T. Huan and H. P. H. Anh. Stable gait optimization for small-sized humanoid robot using modified differential evolution (mde) algorithm. Special Issue of Measurement-Control and Automation Journal, 21, (1), (2018), pp. 63-74. (in Vietnamese).

[13] N. N. Son, H. P. H. Anh, and T. D. Chau. Inverse kinematics solution for robot manipulator based on adaptive MIMO neural network model optimized by hybrid differential evolution algorithm. In Proceedings of the 2014 IEEE International Conference on Robotics and Biomimetics (ROBIO). IEEE, (2014), pp. 2019-2024.

[14] N. Shafii, L. P. Reis, and N. Lau. Biped walking using coronal and sagittal movements based on truncated fourier series. In RoboCup-2010: Robot Soccer World Cup XIII. Springer, (2011), pp. 324-335.

[15] E. Yazdi, V. Azizi, and A. T. Haghighat. Evolution of biped locomotion using bees algorithm, based on truncated Fourier series. In Proceedings of the World Congress on Engineering and Computer Science. Citeseer, (2010), pp. 378-382.

[16] Y. Farzaneh, A. Akbarzadeh, and A. A. Akbari. Online bio-inspired trajectory generation of seven-link biped robot based on T-S fuzzy system. Applied Soft Computing, 14, (2014), pp. 167180. https://doi.org/10.1016/j.asoc.2013.05.013.

[17] D. Gong, J. Yan, and G. Zuo. A review of gait optimization based on evolutionary computation. Applied Computational Intelligence and Soft Computing, 2010, (2010), pp. 1-12. https://doi.org/10.1155/2010/413179.

[18] P. H. Anh. An evolutionary-based optimization algorithm for truss sizing design. Vietnam Journal of Mechanics, 38, (4), (2016), pp. 307-317. https://doi.org/10.15625/0866-7136/7476.

[19] C.-F. Juang and Y.-T. Yeh. Multiobjective evolution of biped robot gaits using advanced continuous ant-colony optimized recurrent neural networks. IEEE Transactions on Cybernetics, 48, (6), (2018), pp. 1910-1922. https://doi.org/10.1109/tcyb.2017.2718037.

[20] B. H. Le and T. M. Thuy. Optimal design for eigen-frequencies of a longitudinal bar using Pontryagin's maximum principle considering the influence of concentrated mass. Vietnam Journal of Mechanics, 39, (1), (2017), pp. 1-12. https://doi.org/10.15625/0866-7136/6058.

[21] T. T. Huan and H. P. H. Anh. Implementation of novel stable walking method for smallsized biped robot. In Proceedings The 8th Viet Nam Conference on Mechatronics (VCM), (2016), pp. 283-292.

[22] R. A. Formato. Central force optimization: A new metaheuristic with applications in applied electromagnetics. In Progress in Electromagnetics Research (PIER), Vol. 77, (2007), pp. 425-491. 